\title{
\#Safe Mapping Platform:A GIS Mobile Crowd Sensing Platform for COVID-19 Self-Tracking and Self-Risk Managing
}

\author{
Homhuan, S., Suwanprasit, C., Namwong, C., Khamnoi, P., Boonma, R., Mate, T. and \\ Wanginkhom, $\mathbf{N}^{1}$ \\ Department of Geography, Faculty of Social Sciences, Chiang Mai University, 239 Huay Keaw Road, \\ Mueang, Chiang Mai, 50200, Thailand, E-mail: chanida.suwanprasit@cmu.ac.th
}

DOI: https://doi.org/10.52939/ijg.v17i5.2009

\begin{abstract}
Increases in the social sector of open data and online mapping technologies are starting new chances for interactive mapping in many research applications. Mobile crowd sensing is an application that gathers data from a network of conscientious volunteers and implements it for a public benefit which is very helpful for collecting related information during the COVID-19 situation. The paper aims to demonstrate the concept of \#Safe Mapping Platform which followed a framework of opensource technology and implementation aspects. The \#Safe Mapping Platform was established for self-tracking and self-risk managing by integrating GIS opensource technologies, location-based services, and LINE application. The developed platform can be adapted to the public for self-tracking and self-risk managing in any health issues in the future.
\end{abstract}

\section{Introduction}

The rapidly spreading of SARS-CoV-2 makes the control COVID-19 inability. The adoption of strict rules to decrease individual mobility and increase social distancing, accompanied by the use of personal protective measures such as masks and gloves has been approached. Thailand was an early country that was affected by SARS-CoV-2. By end of March, there were 1,524 total cases reported (Control, 2020). People have been panicking and worrying about the pandemic situation at that time. Franch-Pardo et a., (2020) reviewed 63 scientific articles for the role of GIS and Spatial analysis in COVID-19. They categorized the articles into 5 general groups including spatiotemporal analysis and disease mapping, health and social geography, environmental variables, data mining, and webbased mapping. However, individual tracking and risk assessment articles have not been much published until now.

Mobile crowd sensing is a concept boosted by reality as these days almost everyone owns smart, wearable, mobile devices equipped with diverse sensors and able to record, process, and transmit a great variety of data that can be stored, shared, and analyzed to improve the knowledge about the phenomena of common and personal benefit for increasing numerous aspects of general well-being (Farkas et al., 2015, He et al., 2020, Higuchi et al., 2014 and Jovanovic et al., 2019). Mobile crowd sensing is a strong paradigm for practically the same problem as participatory sensing. Location tracking and collection of spatial data are the concepts of crowd sensing which engaged people to be a sensor.

Like online mapping applications have become well-known platform users accessing mapping services through their smartphone. Spatial data and mapping are also widespread in social media, with users sharing and tagging geolocated media through various crowdsourcing applications. The potential advantages of using web mapping tools to integrate socio-economic data into global and national platforms could be substantial in terms of facilitating research and allowing the public to compare and contrast locations across the world using a range of indicators. The ability to use online mapping and epidemiology to address emerging population health issues in our society are key components of the descriptive, predictive, and prescriptive stages of combating COVID- 19 .

A hybrid cloud is a cloud computing environment that uses a mix of private cloud and public cloud services with composition between the two platforms (Helmi et al., 2018). LINE is one of the most common social network applications in Thailand. The Messaging API in LINE application lets us develop two-way communication between our services and LINE users. Reply messages are messages that LINE bot sends in response to users' 
messages (LINE_Corporation, 2020). Therefore, the integration between LINE and GIS web applications is very interesting to provide self-tracking and risk managing during the COVID-19 pandemic spreading situation.

This paper aimed to develop and demonstrate a hybrid cloud GIS framework integration with LINE application platform and mobile crowd sensing for both public and self-tracking and risk managing during the pandemic period which hopes to be a benchmark for further related applications in the future.

\section{System Function Designs and Implementation}

The development aimed to help our students in the Department of Geography, Faculty of Social Sciences, Chiang Mai University, Thailand to do tracking, risk managing, and monitoring themselves as the university was closed because of the lockdown policy. But once the platform was distributed to the public, many people were willing to follow and use it for their self-tracking.

The \#Safe Mapping Platform was designed and developed following a prototyping approach to integrating LINE application and GIS web using HTML5/JavaScript techniques which have been developed under opensource and cloud mapping frameworks. The designation of the platform is very simple and easy to use. The platform was developed via opensource including PostgresSQL-PostGIS, NodeJS, Geoserver, LeafletJS, Dialogflow API, Apexcharts, and LINE Bot.

The workflow of the platform is shown in Figure 1. The Open API from the Department of Disease Control, Thailand has been central to the release of COVID-19 daily found cases. It is one of the public sector datasets for free in standardized and shareable form (Department of Disease Control, 2020). Once the user allows using the location, the $\mathrm{x}, \mathrm{y}$ coordinates will be collected and stored in a spatial database. The user ID was transformed to pseudocode to prevent personal information and user location was stored with timestamp data and then displayed in density format. The spatial database not only collection user location but also stores spatial data such as province boundary to show pandemic statistics across the county. An automatic message is generated from Dialog Flow which is a natural language processing platform created by Google widely used for the social network application.

While the user requests to display the tracking map in LINE application, the tracking data will be loaded as a web service through NodeJS with Geoserver and display the map using LeafletJS together with Apexchart. If the user needs any help while using the platform, the user can send a message through LINE Bot which is an automatic response sending back messages directly to the user.

\section{3. \#Safe Mapping Platform Description}

The procedure of the platform was followed the client-server model. The user interface can be categorized into 5 sections including the register section, tracking section, statistics section, and sharing section (Figure 2). To initiate the platform, firstly, the user needs to have a LINE ID which will be a source of data collecting as a mobile crowd sensing. Afterward, users can register for using the platform through QR code named "\#Safe" for LINE friend adding. In the starting interface, the registration form (Figure 3) will available which requires only allowance location, occupation, birth date, sex, and assessment of COVID-19 risk.

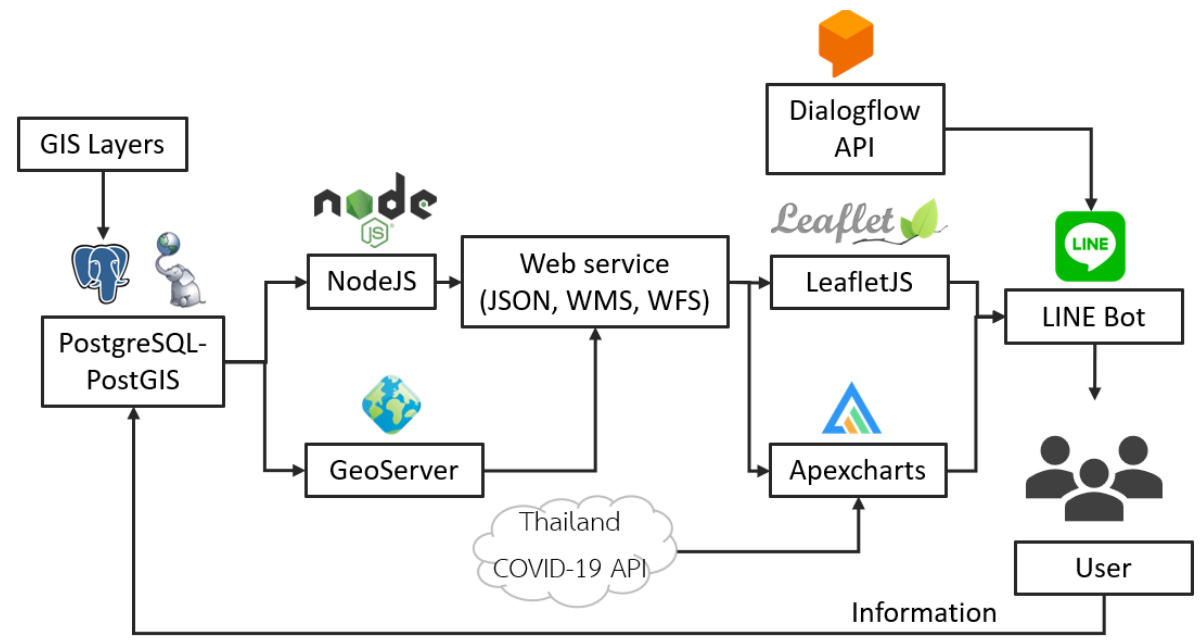

Figure 1: Conceptual framework of \#Safe Mapping Platform 


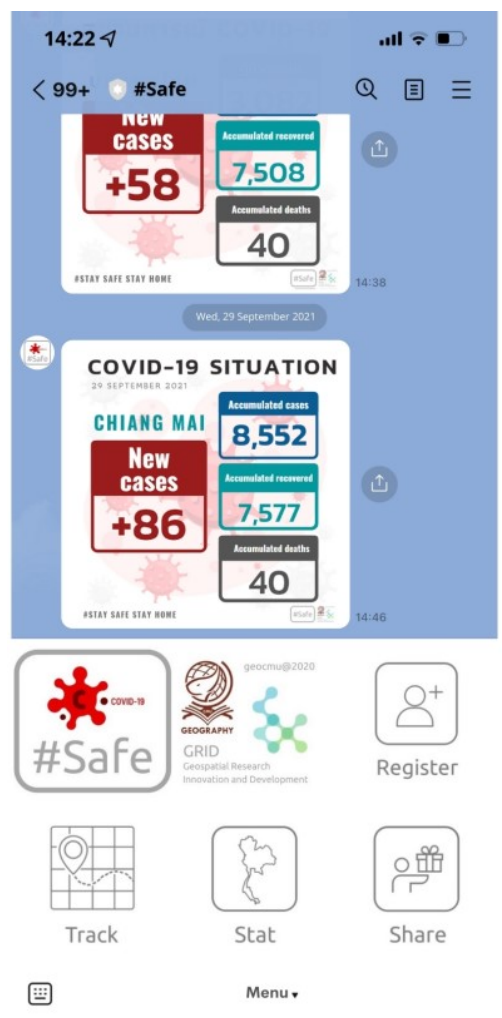

Figure 2: LINE Interface

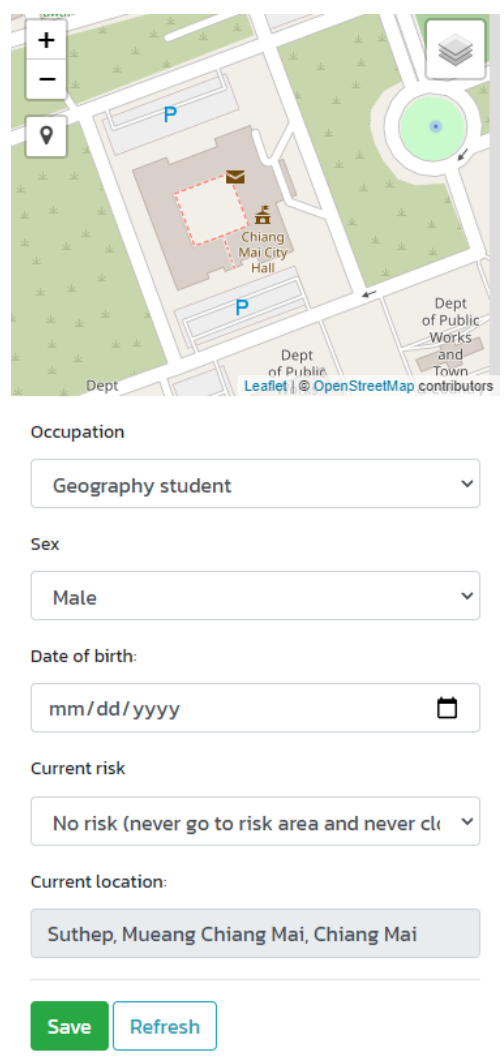

Figure 3: Register Interface

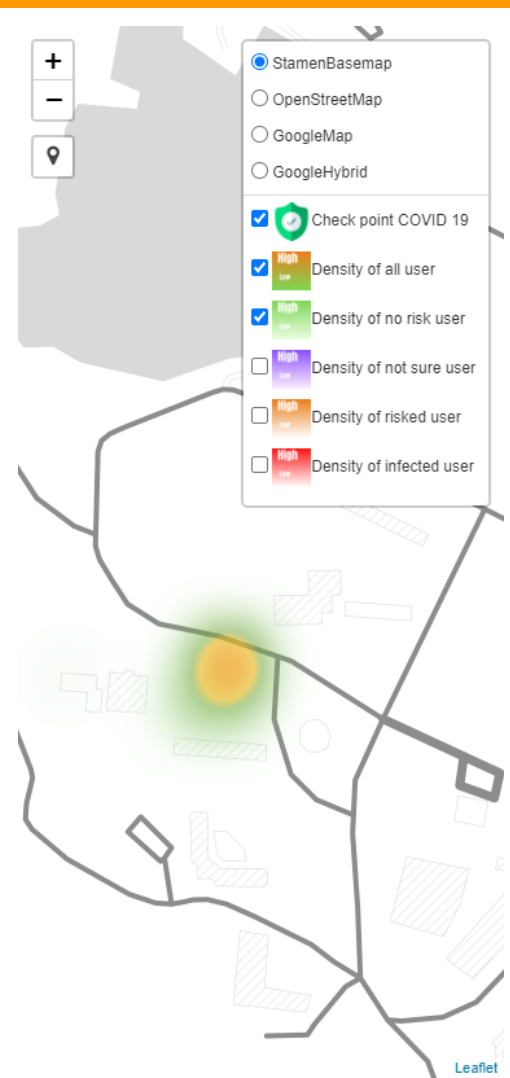

Figure 4: Tracking Interface

The risk level will be observed by the user and can be changed whenever the risk is altered. There are 4 risk levels including No risk (never go to risk area and never close to risked/infected people), Not sure (has some symptoms but never go to risk area or close to risked/infected people), Risked (go to risk area or close to the risk/infected people), and Infected (confirm by a doctor).

- $\quad$ Tracking section

After user allows the platform to access the location on mobile device, user can select layer to get the movement of 4 risk level categories from all users or get the selftracking which is displayed in heatmap format through online mapping (Figure 4).

- $\quad$ Statistics section

In this section, the user allows discovering the statistics of reported cases for the whole of Thailand via online color plate mapping. There are some display graphs along with the online map such as the number of daily cases, number of cases divided by age, and number of cases in each province (Figure 5). 
- $\quad$ Sharing section

Users can provide some useful information to others through pinning the location which offers not only free or low-cost stuff such as alcohol, gel alcohol, grove, and mask but also COVID-19 checkpoints (Figure 6).

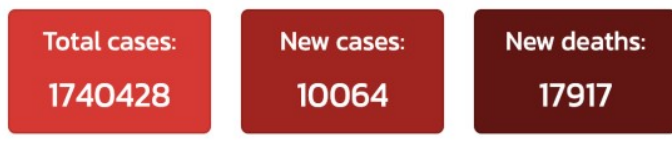

Update: 2021-10-13 07:06:34
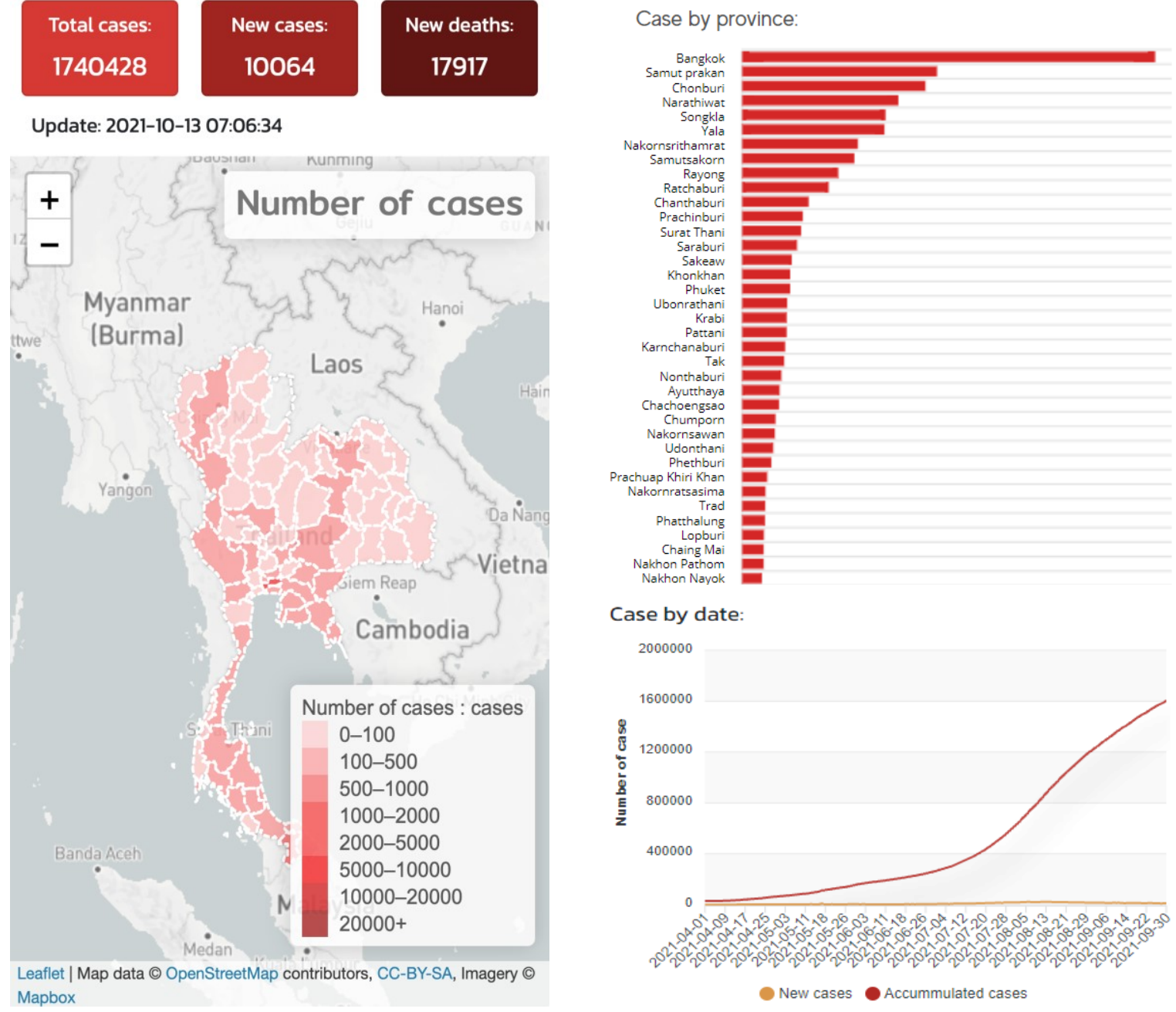

Figure 5: Statistics Interface 


\section{Sharing}

Move the pins to add locations such as checkpoints or masks, alcohol and food sharing locations.

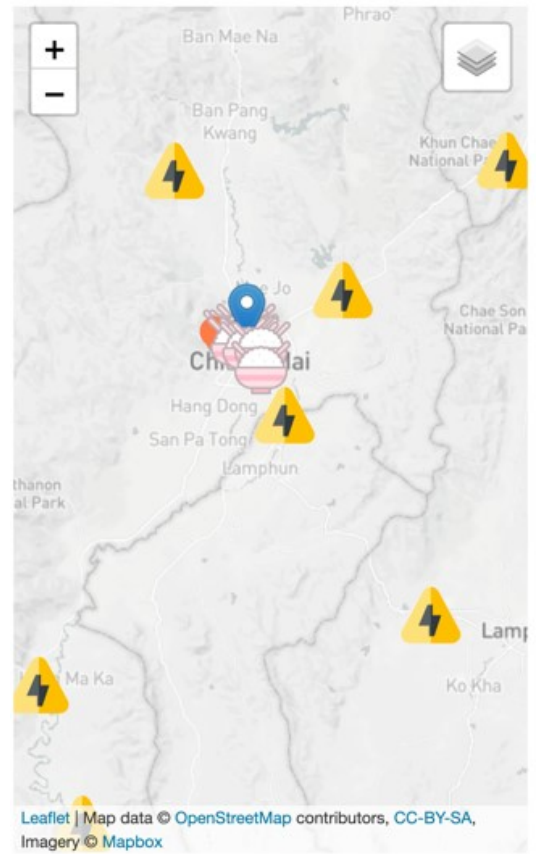

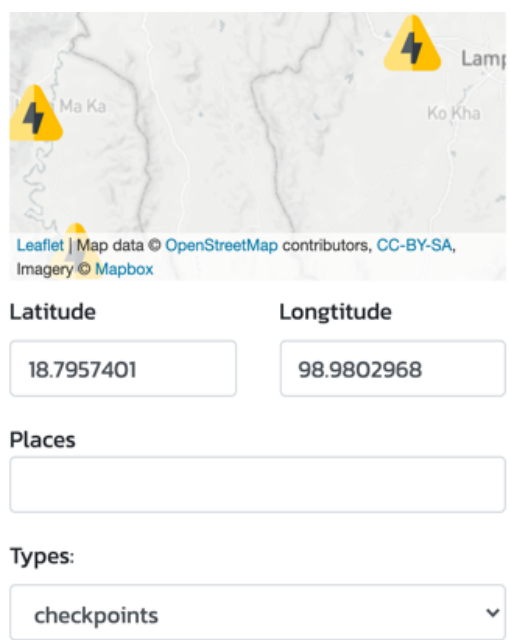

More...

Picture

Choose File No file chosen

\section{Send Edit Delete marker}

Figure 6: Sharing Interface

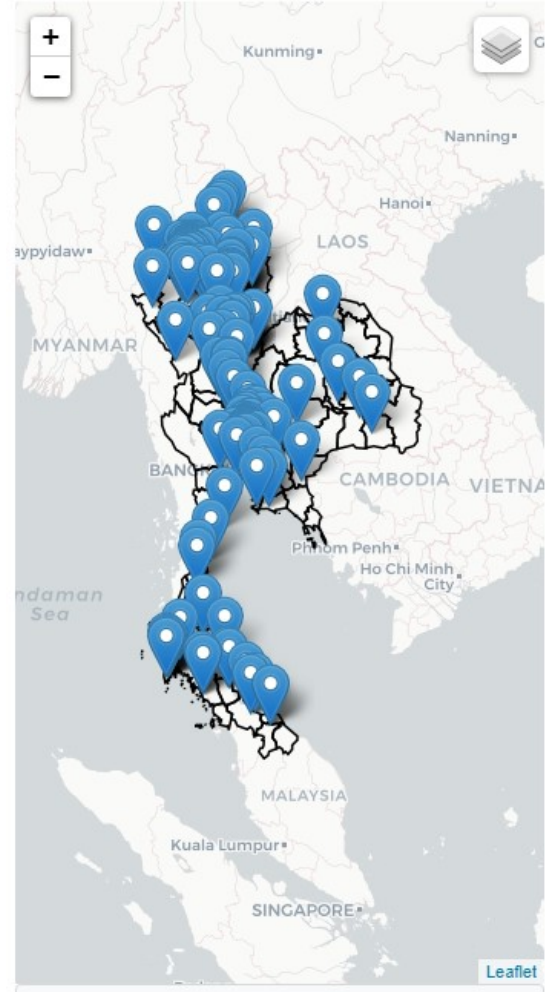

Figure 7: User distribution

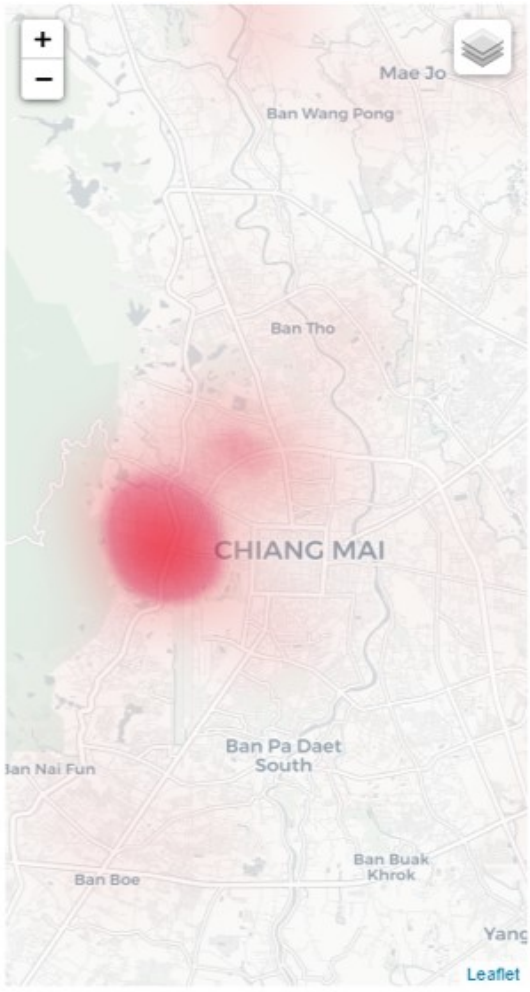

Figure 8: User heatmap distribution 


\section{User Experiences}

There are 389 registered users in LINE application and Figure 7 shows the distribution of the users which are spread over our country and Figure 8 shows a heatmap or density of all users. An online survey was collected to get feedback from our users. The designation, easiness, usefulness information, security, and overall feedback were responded from the users. The experiences of responded user showed that almost user has high and very high satisfied on those issues and requested to continue to launch the platform until the COVID-19 situation is over. The platform has several characteristics that distinguish it from others, such as the requirement that it be launched when in use, which protects users from unauthorized tracking. Furthermore, the user interface and map presentation are convenient and straightforward to interpret, and it is developed with open-source technologies that are free and functionality.

\section{Conclusion}

Mobile crowd sensing, GIS technology, social media, and location-based services are advanced at real-time tracking and displaying in several applications. Although there was a google timeline that tracks only individual users which is not helping to reduce user's risk as it did not show other user locations. By increasing crowd sensing input data, it will help the government to reduce ingestion of patient timeline data.

Moreover, an open-source online mapping platform gives users and developers more flexibility and allows them to work in more valuable. These advantages have moved open source into the mainstream of GIS use. The \#Safe Mapping Platform is one example of a perfectional integration platform that applied those technologies followed an opensource framework for COVID-19 both in public and self-tracking and risk-managing purposes which can play a major role during the COVID-19 pandemic situation. Information from communities or crowded sensing responding to the occurrence is a powerful dataset that can be used for surveillance and confinement to reduce the pandemic of the coronavirus. As a result, this platform might be a prototype for combining several technologies in the future. Since the platform does not run in the background system. Therefore, continuous running of the application must be requested while tracking. Further studies may develop in a native application or add more features such as checkpoints for vaccinating or other useful features.

\section{Acknowledgments}

The authors wish to gratefully acknowledge web hosting support from the Department of Geography, Faculty of Social Sciences, Chiang Mai University.

\section{References}

Control, D. o. D., 2020, Corona Virus Disease (COVID-19). Retrieved from https://ddc.moph.go.th/viralpneumonia/index.php.

Department of Disease Control, 2020, Open API for Developer. Retrieved from https://covid19.ddc.moph.go.th/en/api.

Farkas, K., Feher, G., Benczur, A. and Sidlo, C., 2015, Crowdsending Based Public Transport Information Service in Smart Cities. Ieee Communications Magazine, Vol. 53(8), 158165. doi:Doi 10.1109/Mcom.2015.7180523.

Franch-Pardo, I., Napoletano, B. M., Rosete-Verges, F. and Billa, L.,2020, Spatial Analysis and GIS in the Study of COVID-19. A Review. Science of the Total Environment, Vol. 739(15), 1-36, doi:https://doi.org/10.1016/j.scitotenv.2020.1400 33

He, X. Y., Liu, M. and Yang, G. S., 2020, Spatiotemporal Opportunistic Transmission for Mobile Crowd Sensing Networks. Personal and Ubiquitous Computing. doi:10.1007/s00779020-01439-7.

Helmi, A. M., Farhan, M. S. and Nasr, M. M., 2018, A Framework for Integrating Geospatial Information Systems and Hybrid Cloud Computing. Computers \& Electrical Engineering, Vol. 67, 145-158. doi:10.1016/j.compeleceng.2018.03.027.

Higuchi, T., Yamaguchi, H. and Higashino, T., 2014, Context-supported Local Crowd Mapping via Collaborative Sensing with Mobile Phones. Pervasive and Mobile Computing, Vol. 13, 2651. doi:10.1016/j.pmcj.2013.10.012.

Jovanovic, S., Jovanovic, M., Skoric, T., Jokic, S., Milovanovic, B., Katzis, K. and Bajic, D., 2019, A Mobile Crowd Sensing Application for Hypertensive Patients. Sensors, Vol. 19(2). doi:ARTN 40010.3390/s19020400.

LINE_Corporation, 2020, Messaging API. Retrieved from https://developers.line.biz/en/services/messaging-api/. 